\title{
Primary Myxoid Liposarcoma of the Lower Back in an Adult Female Patient: An Extremely Rare Presentation
}

\author{
Amanda R. Zuback ${ }^{\mathrm{a}, \mathrm{c}}$, Sadaf Qazi ${ }^{\mathrm{b}}$, Jamshed Zuberi ${ }^{\mathrm{a}}$
}

\begin{abstract}
Myxoid liposarcoma is the second most common type of liposarcoma that typically presents in the lower extremities and has a predilection for men. Manifestation of this tumor in the lower back is rare. We report the first known case of an adult woman presenting with a soft tissue mass in her lower back. Following excision and further workup, the mass was positive for rearrangements of the DDIT3 (CHOP) gene at the 12q13 locus, consistent with the diagnosis of a myxoid liposarcoma. The tumor was noted to be intramuscular and extended into the margins, requiring re-excision at the previous resection site. Biopsy of the re-excised area was negative for evidence of residual myxoid liposarcoma. We report this case to highlight the importance of considering myxoid liposarcoma as a differential diagnosis in soft tissue masses presenting in the lower back.
\end{abstract}

Keywords: Myxoid; Liposarcoma; Lower back; Case report

\section{Introduction}

Soft tissue sarcomas are relatively rare malignant tumors of mesenchymal origin, representing as little as $1 \%$ of all malignant neoplasms. The most common type of soft tissue sarcoma is a liposarcoma that consists of four different subtypes [1]. Myxoid liposarcomas are the second most common type of liposarcoma that frequently occur in the fourth to fifth decade of life with a propensity to present in men [1-3]. The most prevalent area for this subtype of liposarcomas to develop is in the lower extremities [1]. Liposarcomas rarely present in the back [4]. We present a case of a 29-year-old woman found to have a myxoid liposarcoma located in her right lower back.

Manuscript submitted August 1, 2020, accepted August 7, 2020

Published online September 23, 2020

aSt. Joseph's Regional Medical Center, 703 Main St, Paterson, NJ 07503, USA bBayonne Medical Center, Carepoint Health, 29 E 29th St, Bayonne, NJ 07002, USA

${ }^{\mathrm{c}}$ Corresponding Author: Amanda R. Zuback, St. Joseph's Regional Medical Center, 703 Main St, Paterson, NJ 07503, USA. Email: arzubes@gmail.com

doi: https://doi.org/10.14740/jmc3558

\section{Case Report}

A 29-year-old woman presented to the hospital for an elective excision on a soft, mobile mass located on her right lower back. The patient stated that the mass had been causing her discomfort and had been present for quite some time. The mass was excised and during the procedure was noted to be intramuscular. Upon biopsy results, the gross specimen was partially capsulated measuring $11.0 \times 10.0 \times 5.5 \mathrm{~cm}$, tan-pink to brown in color with a smooth outer surface. The tumor extended into the margins of the excision. The mass consisted of mainly hypocellular, vaguely lobulated, proliferation of small ovoid to spindle cell admixed with scattered lipoblasts, in a background of abundant myxoid material and arborizing vascularity. At the periphery of the lobules, increased cellularity was noted with moderately increased cytological atypia. Mitotic activity was very rare and areas of round cell composed less than $5 \%$ of the total tumor. In many of the tumor cells, stain with p53 showed evidence of p53 overexpression. Stain with Ki-67 showed a proliferation index of 2-8\%. Fluorescence in situ hybridization (FISH) analysis was positive for detection of rearrangements of the DDIT3 (CHOP) gene at the $12 \mathrm{q} 13$ locus, consistent with the diagnosis of myxoid liposarcoma. Following the diagnosis, the patient had a computed tomography (CT) scan of the chest, abdomen and pelvis, which revealed a resection scar along the right paraspinal musculature with no evidence of lymphadenopathy, lesions or pathology. Due to evidence of the tumor extension into the margins, the patient underwent re-excision at the previous resection site of the mass. The patient tolerated the procedure well and will be followed up for subsequent post-operative visits. The biopsy results of the reexcision revealed no evidence of residual myxoid liposarcoma.

\section{Discussion}

Liposarcomas are one of the most common types of soft tissue sarcomas. According to the World Health Organization, there are four types. These subtypes are myxoid/round cell, pleomorphic, well differentiated and dedifferentiated $[3,5]$. Of the four subtypes, myxoid liposarcomas account for about $20 \%$. Myxoid liposarcomas typically present as a painless slow growing mass [6]. They are frequently located in the intramuscular fascia, under or on muscle [4].

The most common area for myxoid/round cell liposarcomas to develop is in the lower extremities, which make up about $75-80 \%$ of cases. More specifically, about two-thirds of 
cases manifest in the thigh $[1,3]$. In about $8 \%$ of cases they present in the retroperitoneum and 5\% appear in the upper extremities [1]. It is atypical for a liposarcoma to present in the back [4]. To the best of our knowledge, no cases of primary myxoid liposarcomas subtype located in the lower back have been reported. We report a rare case of a myxoid liposarcoma presenting in the right lower back.

Histologically, this subtype exists on a spectrum with variable amounts of round cells. The existence of greater than $5 \%$ of round cells within the mass is associated with a poor prognosis and is considered high-grade $[1,7]$. Approximately $95 \%$ of cases of myxoid/round cell liposarcoma subtype have a $T L S$-CHOP fusion gene on cytogenetic analysis $[7,8]$. This fusion gene is specific for this subtype and is the result of $\mathrm{t}(12 ; 16)(\mathrm{q} 13 ; \mathrm{p} 11)$ translocation. It has been suggested to be a dysfunctional transcriptional regulator that interrupts differentiation of adipocytes resulting in proliferation [8, 9]. In our case, the mass was found to have this specific fusion gene. Seen in less than $5 \%$ of cases, EWS-CHOP fusion gene is a rare variant that has also been observed $[8,9]$.

The standard of care for patient with local disease is wide local surgical resection with the confirmation of negative margins $[10,11]$. Low-grade myxoid liposarcomas have a low local recurrence rate. At a 5-year follow-up, approximately $8 \%$ of diagnosed patients will have local disease recurrence [7]. In comparison to other types of soft tissue sarcomas, myxoid liposarcoma is more radiosensitive [11]. Adjuvant radiation is typically considered for patients who have considerable number of round cells composing the mass [7].

Myxoid/round cell liposarcoma has a tendency to metastasize to extrapulmonary sites, specifically, soft tissue and skeletal sites such as the mediastinum and the retroperitoneum [12, 13]. With the potential to metastasize to distant sites, prompt diagnosis as well as providing the best possible treatment options is critical [14].

\section{Conclusions}

This case illustrates the challenges associated with the diagnosis and management of these rare tumors. Myxoid liposarcomas should be included as a possible differential diagnosis in cases of soft tissue masses that present in the lower back. An early accurate diagnosis will not only result in appropriate treatment from the get-go, but also save both the patient and clinician valuable time and resources. Although a rare entity, considering myxoid liposarcoma in the diagnosis of soft tissue masses may prevent misdiagnosis and possibly alter the clinical approach.

\section{Acknowledgments}

None to declare.

\section{Financial Disclosure}

None to declare.

\section{Conflict of Interest}

None to declare.

\section{Informed Consent}

Not applicable.

\section{Author Contributions}

SQ participated in the case. ARZ wrote the case report. SQ, JZ and $A Z$ revised and edited the case report.

\section{Data Availability}

The authors declare that data supporting the findings of this study are available within the article.

\section{References}

1. Mujtaba B, Wang F, Taher A, Aslam R, Madewell JE, Nassar S. Myxoid liposarcoma with skeletal metastases: pathophysiology and imaging characteristics. Curr Probl Diagn Radiol. 2019.

2. Boyd CJ, Davis C, Kurapati S, Ananthasekar S, Andino DMA, Kilic A. Recurrent myxoid liposarcoma of the hand. Radiol Case Rep. 2020;15(2):150-153.

3. Asano N, Susa M, Hosaka S, Nakayama R, Kobayashi E, Takeuchi K, Horiuchi K, et al. Metastatic patterns of myxoid/round cell liposarcoma: a review of a 25 -year experience. Sarcoma. 2012;2012:345161.

4. Lee EJ, Jeong TJ, Cho HR, Lew BL, Sim WY. A liposarcoma of the chest wall and back. J Am Acad Dermatol. 2011;64(6):1202-1203.

5. Haniball J, Sumathi VP, Kindblom LG, Abudu A, Carter SR, Tillman RM, Jeys L, et al. Prognostic factors and metastatic patterns in primary myxoid/round-cell liposarcoma. Sarcoma. 2011;2011:538085.

6. Crombe A, Loarer FL, Alberti N, Buy X, Stoeckle E, Cousin S, Toulmonde M, et al. Homogeneous myxoid liposarcomas mimicking cysts on MRI: A challenging diagnosis. Eur J Radiol. 2018;102:41-48.

7. Crago AM, Dickson MA. Liposarcoma: Multimodality Management and Future Targeted Therapies. Surg Oncol Clin N Am. 2016;25(4):761-773.

8. Antonescu CR, Tschernyavsky SJ, Decuseara R, Leung DH, Woodruff JM, Brennan MF, Bridge JA, et al. Prognostic impact of P53 status, TLS-CHOP fusion transcript structure, and histological grade in myxoid liposarcoma: a molecular and clinicopathologic study of 82 cases. Clin Cancer Res. 2001;7(12):3977-3987.

9. Antonescu CR, Elahi A, Healey JH, Brennan MF, Lui MY, Lewis J, Jhanwar SC, et al. Monoclonality of multifocal myxoid liposarcoma: confirmation by analysis of 
TLS-CHOP or EWS-CHOP rearrangements. Clin Cancer Res. 2000;6(7):2788-2793.

10. Hoffman A, Ghadimi MP, Demicco EG, Creighton CJ, Torres K, Colombo C, Peng T, et al. Localized and metastatic myxoid/round cell liposarcoma: clinical and molecular observations. Cancer. 2013;119(10):18681877.

11. Kim DW, Jee YS. Solitary metastasis of myxoid liposarcoma from the thigh to intraperitoneum: a case report. World J Surg Oncol. 2019;17(1):172.

12. Noble JL, Moskovic E, Fisher C, Judson I. Imaging of skeletal metastases in myxoid liposarcoma. Sarcoma. 2010;2010:262361.

13. Stevenson JD, Watson JJ, Cool P, Cribb GL, Jenkins JP, Leahy M, Gregory JJ. Whole-body magnetic resonance imaging in myxoid liposarcoma: A useful adjunct for the detection of extra-pulmonary metastatic disease. Eur J Surg Oncol. 2016;42(4):574-580.

14. Bekers EM, Song W, Suurmeijer AJ, Bonenkamp JJ, van der Geest IC, Braam PM, Ploegmakers MJ, et al. Myxoid liposarcoma of the foot: a study of 8 cases. Ann Diagn Pathol. 2016;25:37-41. 